\title{
Artificial Box C/D RNAs Affect Pre-mRNA Maturation in Human Cells
}

\author{
Grigoriy A. Stepanov, Dmitry V. Semenov, Anna V. Savelyeva, Elena V. Kuligina, \\ Olga A. Koval, Igor V. Rabinov, and Vladimir A. Richter
}

Institute of Chemical Biology and Fundamental Medicine, Siberian Branch, Russian Academy of Sciences, Lavrentiev Avenue 8, Novosibirsk 630090, Russia

Correspondence should be addressed to Grigoriy A. Stepanov; stepanovga@niboch.nsc.ru

Received 31 October 2012; Revised 27 January 2013; Accepted 9 February 2013

Academic Editor: Yasushi Okazaki

Copyright (C) 2013 Grigoriy A. Stepanov et al. This is an open access article distributed under the Creative Commons Attribution License, which permits unrestricted use, distribution, and reproduction in any medium, provided the original work is properly cited.

Box C/D small nucleolar RNAs (snoRNAs) are known to guide the $2^{\prime}$-O-ribose methylation of nucleotides in eukaryotic ribosomal RNAs and small nuclear RNAs. Recently snoRNAs are predicted to regulate posttranscriptional modifications of pre-mRNA. To expand understanding of the role of snoRNAs in control of gene expression, in this study we tested the ability of artificial box C/D RNAs to affect the maturation of target pre-mRNA. We found that transfection of artificial box C/D snoRNA analogues directed to HSPA8 pre-mRNAs into human cells induced suppression of the target mRNA expression in a time- and dose-dependent manner. The artificial box C/D RNA directed to the branch point adenosine of the second intron, as well as the analogue directed to the last nucleotide of the second exon of the HSPA 8 pre-mRNA caused the most prominent influence on the level of HSPA8 mRNAs. Neither box D nor the ability to direct $2^{\prime}$-O-methylation of nucleotides in target RNA was essential for the knockdown activity of artificial snoRNAs. Inasmuch as artificial box C/D RNAs decreased viability of transfected human cells, we propose that natural snoRNAs as well as their artificial analogues can influence the maturation of complementary pre-mRNA and can be effective regulators of vital cellular processes.

\section{Introduction}

Small nucleolar RNAs (snoRNA) are commonly known to be involved in the processing of precursor ribosomal RNA (pre-rRNA) and small nuclear RNAs (snRNAs). Box C/D snoRNAs direct 2' -O-methylation of rRNA nucleotides, and box H/ACA snoRNAs guide the conversion of uridine to pseudouridine [1-4]. The snoRNA-dependent modifications are catalyzed by small nucleolar ribonucleoprotein particles (snoRNPs). Box C/D RNAs are associated with four snoRNP core proteins: NOP56, NOP58, fibrillarin, and $15.5 \mathrm{kDa}$ [5-7].

Box C/D RNAs contain the following functional elements: boxes $\mathrm{C}$ and $\mathrm{D}$, which are essential for snoRNA interaction with specific proteins, and a guide sequence that determines the nucleotide to be modified (Figure 1(a)). Some box $\mathrm{C} / \mathrm{D}$ snoRNAs involve a second pair of boxes $\mathrm{C} / \mathrm{D}$, named $\mathrm{C}^{\prime}$ and $\mathrm{D}^{\prime}$, within the snoRNA molecule. In that case, snoRNAs hold two potential guide sequences $[2,4]$.

rRNAs and snRNAs are the main natural targets of $2^{\prime}$-Omethylation that is mediated by box C/D RNAs. However, a number of snoRNA-like transcripts do not have guide sequences that are complementary to rRNA or snRNA, so they have unknown functions and are called "orphan snoRNAs" [8, 9]. Bioinformatics analysis revealed that some of these orphan RNAs were complementary to pre-mRNAs or mature mRNAs [10]. MBII-52 box C/D snoRNA containing a guide sequence complementary to the serotonin receptor $2 \mathrm{C}$ pre-mRNA was reported to take part in controlling the posttranscriptional modification of the target pre-mRNA (conversion A to I) [11]. It was also shown that HBII-52 snoRNA could regulate alternative splicing of the complementary pre-mRNA [12]. 


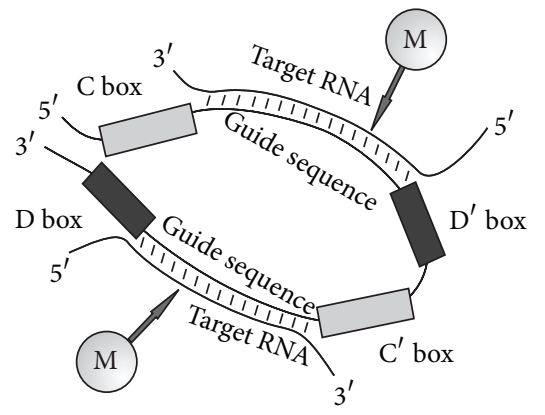

(M) Target nucleotide to be $2^{\prime}$-O-ribose methylated

(a)

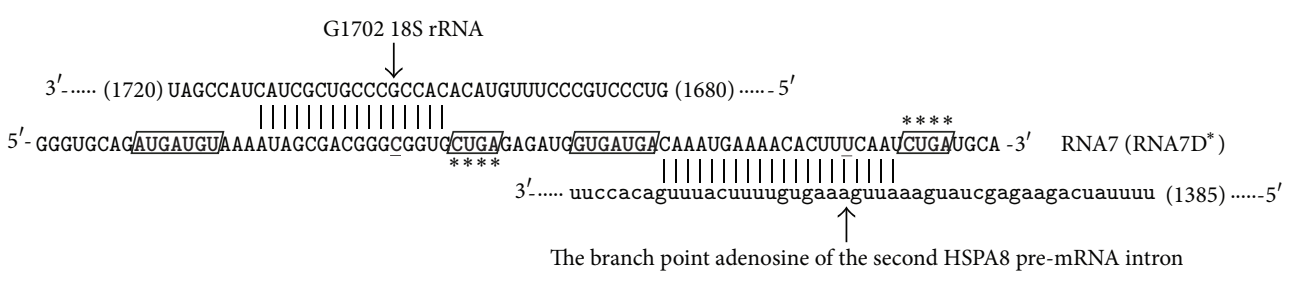

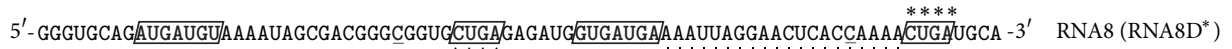

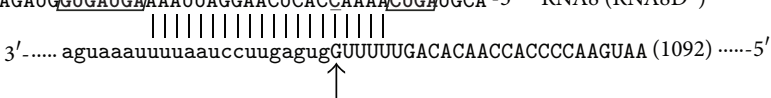

The last nucleotide of the second HSPA8 pre-mRNA exon (the donor site)

5' - GGGUGCACAUGAUGUAAAAUAGCGACGGGCGGUGCUGAGAGAUdGUGAUGAAAAUUAGGAACUCACCAAACUGAUGCA - $3^{\prime}$ RNA9

I $3^{\prime}-\ldots .$. aguaaauuuuaauc cuugagugGUUUUUGGACACAACCACCCCAAGUAA (1092) $\ldots . . .-5^{\prime}$

个

The first nucleotide of the second HSPA8 pre-mRNA intron

$5^{\prime}$ - GGGUGCAGAUGAUGUAAAUAGCGACGGGCGGUGCUGAGAGAUGGUGAGAUACAGACGUUUGGCAUCUGUCUGAJGCA - $3^{\prime}$ RNA10

$\underset{3^{\prime}-\ldots . . . \text { CGCAGGUUAGUCUGCAAACCGUAgacauuuuccacaguuuacuuuugu (1414) } \cdots . . .-5^{\prime}}{\|}$

The first nucleotide of the third HSPA8 pre-mRNA exon (the acceptor site)

$5^{\prime}$ - GGGUGCAGAUGAUGUAAAUAGCGACGGGGGUGCUGAGAGAUGGUGAUGAUCAGACGUUUGGCAUCUGUACUGAUGCA - $3^{\prime}$ RNA11

|l|||||||||||||||||||||

$3^{\prime}$-..... GCAGGUUAGUCUGCAAACCGUAgacauuuuccacaguuuacuuuugug (1415) $\cdots \cdots-5^{\prime}$

个

The last nucleotide of the second HSPA8 pre-mRNA intron

$5^{\prime}$ - GGGUGCAGAUGAUGUAAAUAGCGACGGGCGGUGCUGAGAGAUGGGAUGAACGACGGUCUAAACCCUGAUGCACCC - $3^{\prime}$ RNA4502
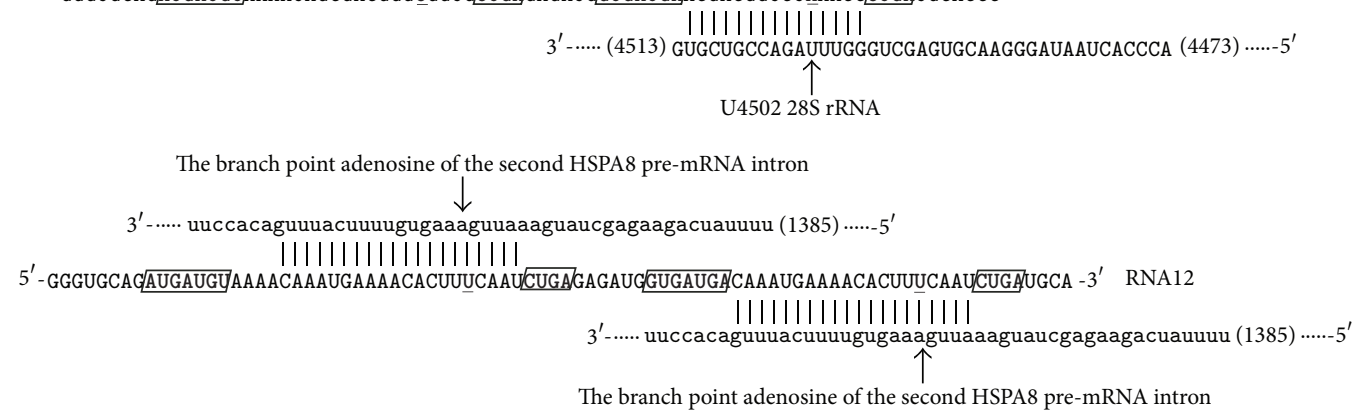

(b)

Figure 1: (a) Schematics of a guide box C/D snoRNA structure and the interaction between a snoRNA and target RNAs. (b) Base-pairing interactions between an artificial box C/D RNA and target RNAs. The arrows indicate potential targets of $2^{\prime}$-O-methylation. Uppercase letters in the target pre-mRNA sequences correspond to exons, and small letters indicate the intron sequences. Boxes $\mathrm{C}$ and $\mathrm{D}$ are indicated as GUGAUGA and CUGA enclosed in rectangles, respectively. The nucleotides to be complementary to target nucleotides are shown as $\underline{\mathbf{C}}$. RNA7D* and RNA8D* were constructed by changing the CUGA sequence in both box D and $\mathrm{D}^{\prime}$ to AAAA. 
In earlier studies, the development of snoRNA expression vectors was one of the main approaches to study the influence of box C/D snoRNAs on target genes in mammalian cells. The developed DNA vectors encoded mature snoRNAs or artificial pre-mRNA fragments that were processed to snoRNA $[1,13]$. Using such an approach, it was shown that introducing sequences complementary to a selected pre-mRNA into box C/D RNA structures allowed knockdown of the target mRNA [13]. In study by Ono and coworkers, such an antisense element was placed into HBII-180C box C/D RNA instead of the structural element referred to as the $\mathrm{M}$ box [13]. The natural $\mathrm{M}$ box is located within the HBII-180C snoRNA molecule and involves box $\mathrm{C}^{\prime}$. Replacing the $\mathrm{M}$ box with sequence complementary to a selected pre-mRNA allowed the snoRNA analogue to be directed to this pre-mRNA and to knockdown the mRNA target. Interestingly, the ability of the box C/D snoRNA to direct 2'-O-methylation of rRNA nucleotides was not essential for the knockdown effect [13].

Therefore, recent results indicate that box C/D RNAs that include a sequence element complementary to a premRNA could control the posttranscriptional maturation of the pre-mRNA, with antisense elements located in different parts of the snoRNA molecules. In our study, we proposed and employed a novel approach to investigating the snoRNA ability to control gene expression. The approach involves direct application of artificial RNA constructions containing structural features of snoRNAs instead of DNA vectors expressing snoRNAs. Such snoRNA analogues can be addressed to different pre-mRNA or mRNA targets and delivered to the cells using transfection methods.

We selected the pre-mRNA of the HSPA8 gene encoding the human heat shock cognate $71 \mathrm{kDa}$ protein (HSC70) as a model target of snoRNA-directed action. Stress-induced proteins related to the heat shock protein 70 (HSP70) family perform numerous functions in the cell, including folding of nascent cellular proteins, regulation of intracellular transport, and directed degradation of proteins. Moreover, heat shock proteins take part in controlling repair systems, the cell cycle, cell proliferation, and differentiation [14-17]. These proteins inhibit cell death processes and cause the survival of tumorigenic cells attacked by stress factors such as cytokines, radiation, oxidative stress, and drugs, which reduces the effects of some therapeutic approaches. Therefore, controlling the expression of heat shock family genes is believed to be an option for developing complex anticancer therapy approaches [18-20].

Earlier, we determined that artificial U24 box C/D snoRNA analogues directed to HSPA 8 pre-mRNA induced partial splicing impairments, namely, skipping of the exon immediately adjacent to targeted intron [21].

In this study, to further estimate the influence of box C/D RNAs analogs directed to HSPA8 pre-mRNA on the maturation of the target pre-mRNA, we synthesized the set of artificial analogues of snoRNA. It was shown that artificial box C/D RNAs directed to HSPA8 pre-mRNA caused not only partial splicing impairments, but also the suppression of the target mRNA expression. It was also found that the transfection of the analogues into MCF-7 human adenocarcinoma cells decreased the viability of the transfected cells.

\section{Materials and Methods}

2.1. Artificial Box C/D RNAs Synthesis. To design artificial box C/D RNA guide sequences, we used the HSPA8 pre-mRNA sequence, chr11:122928200-122932901, from the GRCh37/hg19 human genome assembly and the HSPA8 mRNA sequence from NCBI RefSeq NM_006597.4. In the text, the exons and introns of HSPA 8 pre-mRNA were numerated from the $5^{\prime}$-end to the $3^{\prime}$-end of the pre-mRNA. To identify the branch point adenosine in the second intron of HSPA 8 pre-mRNA, we used the algorithm developed by Kol et al. [22].

Synthetic analogues of box C/D RNAs were obtained via the in vitro transcription of PCR-amplified DNA templates with T7 RNA polymerase (Fermentas, Vilnius, Lithuania). DNA templates were synthesized using the following oligonucleotides:

$$
\begin{aligned}
& \text { U24_Base } 5^{\prime} \text {-TGCAGATGATGTAAAATAGCGA } \\
& \text { CGGGCGGTGCTGAGAGATGGTGATGA-3' } \\
& \text { (a template for DNA7, DNA8, and DNA4502); } \\
& \text { U24_Base_5BP } 5^{\prime} \text {-TGCAGATGATGTAAAACAA } \\
& \text { ATGAAAACACTTTCAATCTGAGAGATGGTG } \\
& \text { ATGA-3' (a template for DNA12); }
\end{aligned}
$$$$
\text { U24-T7 5' -ATGCAGCTAATACGACTCACTATAGG }
$$$$
\text { GTGCAGATGATGTAAAATAG-3' (a universal right }
$$$$
\text { primer for DNA7, DNA8, and DNA4502); }
$$$$
\text { T7_5BP 5'-ATGCAGCTAATACGACTCACTATAGG }
$$$$
\text { GTGCAGATGATGTAAAACAAATG-3' }
$$$$
\text { (a left primer for DNA12); }
$$$$
24.75^{\prime} \text {-TGCATCAGATTGAAAGTGTTTTC }
$$$$
\text { ATTTGTCATCACCATCTCTCAG-3' }
$$$$
\text { (a right primer for DNA7 and DNA12); }
$$$$
24.85^{\prime} \text {-TGCATCAGTTTTGGTGAGTTCCTA }
$$$$
\text { ATTTTCATCACCATCTCTCAG-3' (a right primer }
$$$$
\text { for DNA8); and }
$$$$
\text { 28U4502 5'-GGGTGCATCAGGGTTTA }
$$$$
\text { GACCGTCGTTCATCACCATCTCTCAG-3' }
$$$$
\text { right primer for DNA4502). }
$$

Each of the artificial RNA, namely, RNA7 (79 nt), RNA8 (79 nt), RNA9 (78 nt), RNA10 (79 nt), and RNAll (79 nt), RNA12 (84 nt), RNA7D* (79 nt), RNA8D* (79 nt), RNA4502 $(77 \mathrm{nt})$ were synthesized using corresponding DNA-template (see DNA7, DNA8, etc.).

All oligonucleotides were obtained from LMC ICBFM SB RAS, Novosibirsk. RNA transcripts were purified via ionpair reverse phase high-performance liquid chromatography (RP-HPLC) on a Milichrome A-02 liquid chromatograph using the ProntoSIL-120-5-C18 sorbent and a $2.0 \times 7.5 \mathrm{~mm}$ column (Econova, Novosibirsk, Russia), followed by ethanol precipitation (with $6.0 \mathrm{M} \mathrm{NaOAc}$ ). RNA concentrations were determined spectrophotometrically, and RNA integrity was verified on $1.5 \%$ agarose or $5 \%$ denaturing polyacrylamide gels.

2.2. Transfection of MCF-7 Cells with Synthetic RNAs and Isolation of Total Cellular RNA. MCF-7 cells obtained from the 
Russian Cell Culture Collection of Vertebrates (Institute of Cytology, Russian Academy of Sciences, St. Petersburg, Russia) were cultured in Iscove's modified Dulbecco's medium (IMDM) (Invitrogen, Carlsbad, CA, USA) containing $10 \mathrm{mM}$ L-glutamine, $40 \mu \mathrm{g} / \mathrm{mL}$ gentamicin (Sigma-Aldrich, St Louis, MO, USA) and $10 \%$ fetal bovine serum (FBS) (Biolot, St. Petersburg, Russia).

Cells were seeded at $2 \times 10^{5}$ cells/well in a 6-well plate and incubated $24 \mathrm{~h}$ in a humidified incubator at $37^{\circ} \mathrm{C}$ with $5 \% \mathrm{CO}_{2}$. Synthetic analogues of box C/D RNAs were preincubated with the Lipofectamine reagent (Invitrogen) according to the manufacturer's protocol and were added to the culture medium in the concentration indicated in the figure legends. After incubating the cells with the analogs, total RNA was isolated using the Trizol reagent (Invitrogen) according to the manufacturer's protocol.

\subsection{Accumulation of Radiolabeled Artificial Box C/D RNAs} in Human Cells. Synthetic RNAs were $5^{\prime}-\left[{ }^{32} \mathrm{P}\right]$-labeled with T4 polynucleotide kinase (Biosan, Novosibirsk, Russia). RNA forms were separated on a $10 \%$ denaturing polyacrylamide gel, and individual RNA forms were eluted from gel bands into $0.3 \mathrm{M} \mathrm{NaOAc}$. Transfection of radiolabeled RNAs into human cells was performed as described above. The transfected cells were cultured for time intervals ranging from $3 \mathrm{~h}$ to $72 \mathrm{~h}$. Total RNA was isolated with Trizol and analyzed in a $10 \%$ denaturing polyacrylamide gel followed by autoradiography of radiolabeled RNA fragments.

2.4. Flow Cytometry Analysis. MCF-7 cells $\left(1 \times 10^{6}\right)$ in 6-well plates were transfected with $160 \mathrm{nM}$ FAM-labeled (FAMcarboxyfluorescein) artificial RNA. Control cells were incubated with Lipofectamine only. After incubation for 24 hours, the cells were detached with $0.025 \%$ trypsin and washed twice with IMDM containing 10\% FBS. The transfected cells were scanned using a FACSCanto II flow cytometer (BD Biosciences, Franklin Lakes, NJ, USA) and the data were analyzed by FACSDiva Software (BD Biosciences).

2.5. RT-PCR. The reverse transcription and cDNA amplification (RT-PCR) were performed in the one-tube reaction mixture "Real Best Master Mix RT" (Vektor-Best, Novosibirsk, Russia) with the addition of Sybr Green (Biodye, Moscow, Russia) in the case of real-time RT-PCR. The following gene-specific primers were used: HSPA8: hsp-2.1 $5^{\prime}$-ACTGAACGGTTGATCGGTGA-3' and hsp-8.2 $5^{\prime}$-AGATGAGCACGTTTCTTTCT-3'; HPRT: HPRT_1 $5^{\prime}$ CATCAAAGCACTGAATAGAAAT- $3^{\prime}$ and HPRT_2 $5^{\prime}$-TATCTTCCACAATCAAGACATT-3'; GAPDH: GAPDH-1 5' GAAGATGGTGATGGGATTTC- $3^{\prime}$ and GAPDH-2 $5^{\prime}$ GAAGGTGAAGGTCGGAGT- ${ }^{\prime}$; ; and 28S rRNA: 28-2.1 $5^{\prime}$-TAGACCGTCGTGAGACAGGT-3' and 28-2.2 $5^{\prime}$-ATTGGCTCCTCAGCCAAGCA-3'. To compare PCR product yields, we carried out real-time RT-PCR (on Bio-Rad iQ5 Cycler, Hercules, CA, USA). The data were analyzed using iQ5 system software (Bio-Rad). Mean values $( \pm S D)$ from 3 independent experiments were represented. The PCR products were analyzed in a $4 \%$ denaturing polyacrylamide gel or $1.5 \%$ agarose gel.

2.6. MTT Assay. To estimate the influence of the artificial box C/D RNA on MCF-7 human adenocarcinoma cell viability and proliferation we used MTT assay (MTT (3(4,5-dimethyl-2-thiazolyl)-2,5-diphenyl-2Htetrazolium bromide), Sigma-Aldrich). Aliquots $(150 \mathrm{~mL})$ containing $2.5 \times$ $10^{3}$ cells were plated onto 96-well plates and incubated at $37^{\circ} \mathrm{C}$ with $5 \% \mathrm{CO}_{2}$. After $24 \mathrm{~h}$, the cells were transfected with 40-160 nM artificial RNAs. After incubation for $72 \mathrm{~h}$, MTT was added to the cells at a final concentration of 0.5 $\mathrm{mg} / \mathrm{mL}$ and the plates were incubated at $37^{\circ} \mathrm{C}$ for $3 \mathrm{~h}$. The medium was removed, followed by the addition of $0.15 \mathrm{~mL}$ of DMSO to each well. The plates were read at 570 and $620 \mathrm{~nm}$ using an Apollo LB912 plate reader (Berthold Technologies, Oak Ridge, TN, USA). Cell viability was determined as the absorbance at $570 \mathrm{~nm}$ with reference to $620 \mathrm{~nm}$ and expressed as a percentage of control (control cells were incubated with Lipofectamine only) \pm SD for triplicate independent experiments.

\section{Results and Discussion}

3.1. Structure of Artificial Box C/D RNAs. To investigate the influence of box C/D RNA on pre-mRNA maturation, we constructed and synthesized U24 snoRNA analogues containing guide sequences complementary to HSPA8 premRNA. Human U24 is a 76 nt long conserved RNA which contains two pairs of $\mathrm{C} / \mathrm{D}$ boxes $\left(\mathrm{C}, \mathrm{D}, \mathrm{C}^{\prime}, \mathrm{D}^{\prime}\right)$. It is predicted to guide the $2^{\prime} \mathrm{O}$-ribose methylation of both $28 \mathrm{~S}$ rRNA C2338 and $\mathrm{C} 2352[23,24]$.

Analogues were constructed to direct $2^{\prime}$-O-methylation of G1702 in 18S rRNA and of the HSPA 8 pre-mRNA nucleotides that were critical to splicing the second intron of the pre-mRNA-target (Figure 1(b)). To obtain the artificial box C/D RNAs, we constructed a series of DNA templates and primers containing the $\mathrm{T} 7$ promoter. The templates were amplified, and the PCR-products were used as the templates for in vitro transcription with T7 RNA polymerase.

The artificial RNAs had two pairs of C/D boxes $(C, D$, $\left.\mathrm{C}^{\prime}, \mathrm{D}^{\prime}\right)$ and two guide sequences. The first guide sequence (from the $5^{\prime}$-terminus) was directed to G1702 in 18S rRNA, which was one of the key nucleotides of the ribosomedecoding center [25]. Earlier, we studied the changes in the 18S rRNA structure after transfection of artificial box $\mathrm{C} / \mathrm{D}$ RNAs into human cells. The analysis revealed that artificial RNAs induced the addition of reverse transcription termination sites on rRNA, whereas the snoRNA analogues did not cause $2^{\prime}$-O-methylation of the target nucleotide [21]. The second guide sequence of the artificial RNAs was targeted to the HSPA 8 pre-mRNA nucleotides that were critical to splicing the second intron, namely, the branch point adenosine in the second HSPA8 pre-mRNA intron (RNA7), the last nucleotide of the second exon (RNA8), the first and the last nucleotides of the second intron (RNA9 and RNA11, respectively), and the first nucleotide of the third exon of the target pre-mRNA (RNA10, Figure 1(b)). Artificial box C/D 
RNA designated as RNA12 contained two guide sequences directed to the branch point adenosine in the second HSPA8 pre-mRNA intron (Figure 1(b)). Analogues RNA7D* and RNA8D*, where both the box D and $\mathrm{D}^{\prime}$ CUGA sequences were replaced with AAAA (Figure 1(b)), were targeted to the branch point adenosine in the second intron and the last nucleotide of the second exon of HSPA8 pre-mRNA, respectively.

We have also obtained box C/D RNA analogue directed to two rRNA nucleotides, namely, G1702 in 18S rRNA and U4502 in 28S rRNA (RNA4502, Figure 1(b)). In other studies, we investigated the influence of such analogues on human cells [21]. Here we used RNA4502 as control RNA that was not complementary to the HSPA8 pre-mRNA.

\subsection{Accumulation of Artificial Box C/D RNAs in Transfected} Human Cells. To investigate the efficiency of artificial box C/D RNAs transfection into human cells, we synthesized FAM-labeled RNA analogues and studied the internalization and compartmentalization of the RNAs in MCF-7 cells via fluorescence microscopy. It was found that the fluorescent RNAs were detected in the cytoplasm as well as in nuclei. These results were confirmed with RT-PCR of nuclear and cytoplasmic total RNA from MCF-7 cells transfected with the artificial box C/D RNA [21].

Here, to estimate the efficiency of transfection of box C/D RNA into human cells we treated MCF-7 cells with artificial FAM-labeled RNA in complex with Lipofectamine and analyzed cell populations using flow cytometry. The analysis of cells transfected with FAM-RNA8 revealed that $50 \%$ of the treated cells reliably contained the fluorescent snoRNA analogue (Figure 2(a)). The similar results were obtained for the complete set of box C/D RNA analogues used.

Analysis of radiolabeled box C/D RNA analogue accumulation and integrity in human cells showed that full-length RNA (79 nt long) molecules were detected in the transfected cells at least $72 \mathrm{~h}$ after RNA transfection (in complex with Lipofectamine, Figure 2(b)). Thus, we concluded that artificial box C/D RNAs effectively accumulated and persisted in human cells at least $72 \mathrm{~h}$ under the transfection conditions used.

\subsection{Artificial Box C/D RNAs Affect the Maturation of Comple-} mentary Pre-mRNA. We transfected artificial box C/D RNAs (160 nM RNA complexed with Lipofectamine in culture medium) into MCF-7 human mammary adenocarcinoma cells, isolated total RNA $21 \mathrm{~h}$ after transfection, and performed RT-PCR with primers specific to HSPA8 mRNA. We found that transfection of the artificial analogues into MCF7 human cells suppressed the HSPA8 mRNA level. As shown in Figure 3, the artificial U24 box C/D RNA directed to the branch point adenosine of the second intron of the HSPA8 pre-mRNA (RNA7) and to the last nucleotide of the second exon of the HSPA8 pre-mRNA (RNA8) caused the decrease in target mRNAs level by about $50 \%$ and $40 \%$, respectively. In the same conditions, box C/D RNA analogues directed to other nucleotides in the HSPA8 pre-mRNA (the first and the last nucleotides of the second intron (RNA9 and RNA11, respectively), and the first nucleotide of the third exon of the target pre-mRNA (RNA10, Figure 1(b))) had smaller effects, which were less than $30 \%$.

Interestingly, the insertion of additional complementary elements to the artificial box C/D RNA structure strengthened the suppression effect. Box C/D RNA analogues having two identical guide sequences directed to the branch point adenosine of the second intron of the HSPA 8 pre-mRNA (RNA12, Figure 1(b)) intensified the downregulation effect on the target mRNA level (1.7-2.2 times) compared to the effect from RNA7 (Figure 3).

We next examined the time- and concentrationdependence of HSPA8 mRNA suppression. The higher the concentration of box C/D RNA targeted to the branch point adenosine of the second intron of the HSPA 8 pre-mRNA during transfection, the lower the HSPA8 mRNA level (up to $10 \%$ ) in MCF-7 cells (Figure 4). Increasing the transfection time from $6 \mathrm{~h}$ to $72 \mathrm{~h}$ reduced the HSPA 8 mRNA amount to $30 \%$ of its original level (a 3 -fold decrease) (Figure 5(a)).

The decreased yield of the major product of HSPA8specific RT-PCR was accompanied by increased yield of minor products corresponding to aberrant (shorter) products of HSPA8 pre-mRNA splicing. The primary structures of RT-PCR products were determined with Sanger sequencing and are schematically represented in Figure 5(b). The detected products were determined to be HSPA8 mRNA splicing variants that resulted from skipping the second or simultaneously skipping both the third and fourth exons of the major mRNA isoform. The skipping of exons resulted in a shift in the mRNA reading frame and the appearance of premature stop-codons. It can be supposed that the aberrant splice variants undergo the rapid degradation through the nonsense-mediated mRNA decay pathway $[26,27]$.

The directional influence on the pre-mRNA splicing is one of the promising approaches for gene expression modulation. Such an approach has some advantages; for instance, it provides control of the gene expression in stages of mRNA maturation prior to mRNA appearance in the cytoplasm [28-30]. Thus, our approach implying the use of artificial snoRNA analogues affecting the splicing and overall mRNA isoform levels may be a prospect for the development of novel therapeutic approaches.

\subsection{The Effect of Box C/D RNA Analogues on the Viability} of Human Adenocarcinoma MCF-7 Cells. We also estimated the influence of the artificial box C/D RNA on MCF-7 cell viability and proliferation using the MTT assay. We found that box C/D RNA analogues directed on nucleotides in HSPA8 pre-mRNA decreased MCF-7 viability (Figure 6). Some interrelationship between an ability of a particular snoRNA to decrease HSPA8 mRNA level and its effect on cell viability was found. For instance, the artificial box C/D RNAs directed to the branch point adenosine of the second intron of the HSPA8 pre-mRNA (RNA7, RNA12, and RNA7D $^{*}$ ) caused the most prominent influence on MCF-7 viability and reduced MTT-index by more than $28 \%$ at a final concentration of artificial RNAs $80 \mathrm{nM}$ (Figure 6). As shown 

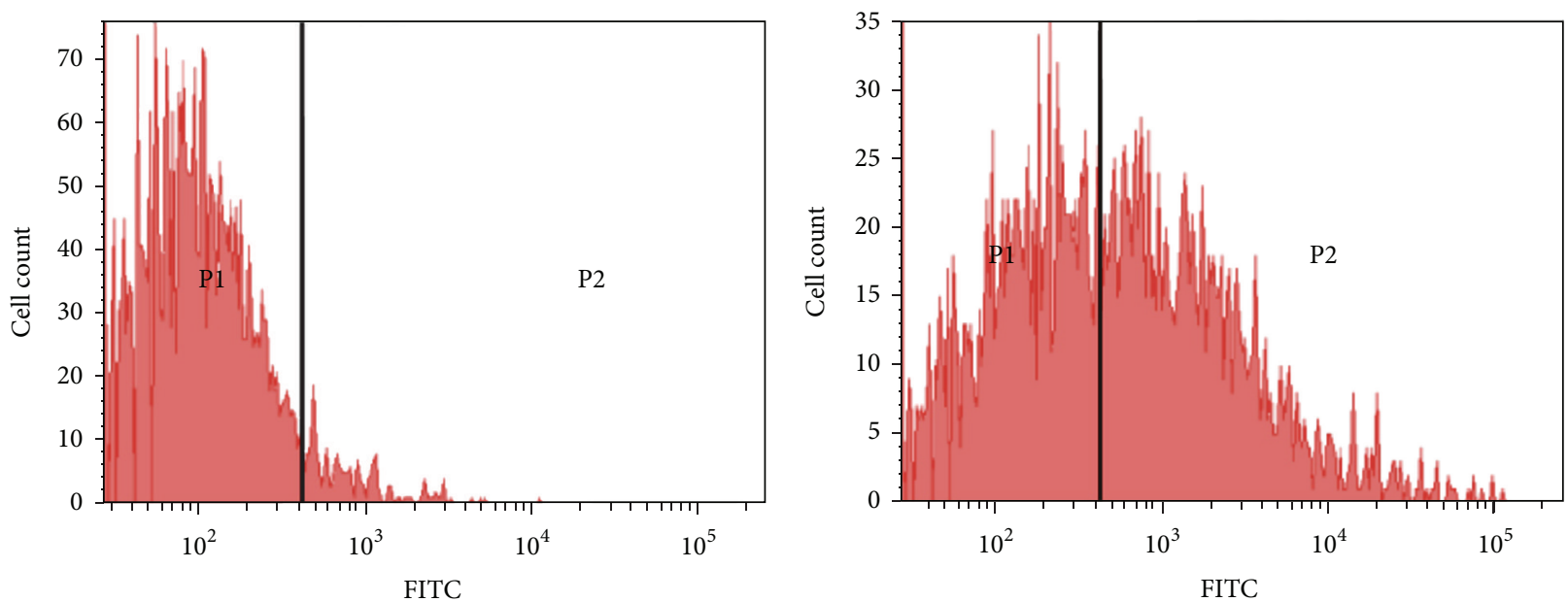

(a)

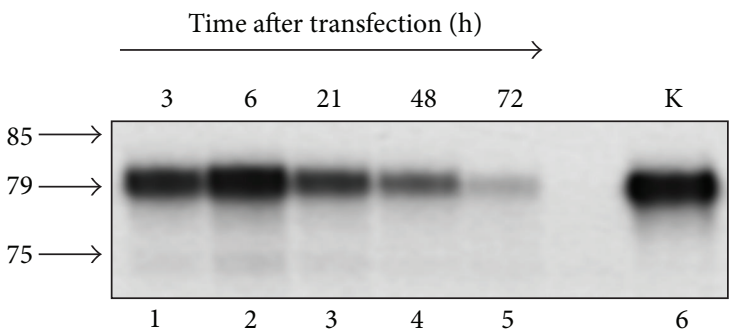

(b)

FIgURE 2: (a) Accumulation of FAM-labeled artificial box C/D RNAs in human cells. MCF-7 cells transfected with FAM-labeled RNA8 analogue using Lipofectamine were analyzed with flow cytometry. "P2" population represents MCF-7 cells positively stained with FAMlabeled RNA, with "P1" population representing nonstained cells. (b) Presence of radiolabeled artificial box C/D RNA in total RNA from transfected MCF-7 cells. Radiolabeled RNA8 was transfected into cells using Lipofectamine, and the cells were cultured for time intervals from $3 \mathrm{~h}$ to $72 \mathrm{~h}$ (lanes 1-5). Total cellular RNA was isolated and analyzed in a 10\% denaturing polyacrylamide gel followed by autoradiography of the radiolabeled RNA fragments. The RNA loading was normalized by spectrometry, and $3 \mu \mathrm{g}$ of total cellular RNA was used per lane. Lane 6 intact radiolabeled artificial box C/D RNA. The arrows indicate marker mobility (in parallel sequencing reaction product were loaded on the same gel).

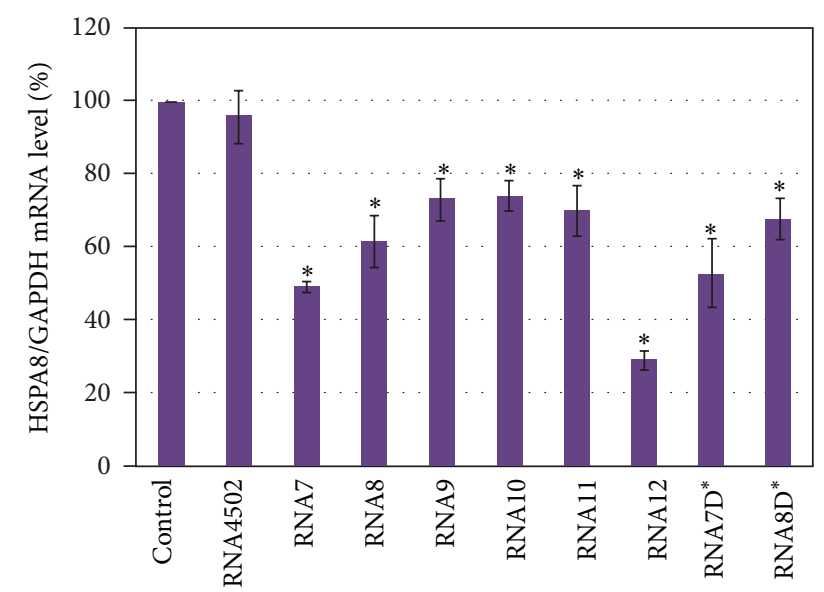

FiguRE 3: Level of HSPA8 mRNA $24 \mathrm{~h}$ after transfection of artificial box C/D RNAs into MCF-7 cells (160 nM RNA in culture medium). MCF-7 human cells were transfected with RNA7, RNA8, RNA9, RNA10, RNA11, RNA12, RNA7D*, RNA8D* directed to HSPA8 pre-mRNA and RNA4502 directed to ribosomal RNAs. Control cells were incubated with Lipofectamine only. The level of mRNA was determined with quantitative RT-PCR and was represented as relative values normalized to the level of GAPDH mRNA. The error bars represent standard deviations. The asterisks $\left(^{*}\right)$ indicate significant difference from control $(P<0.05)$. 

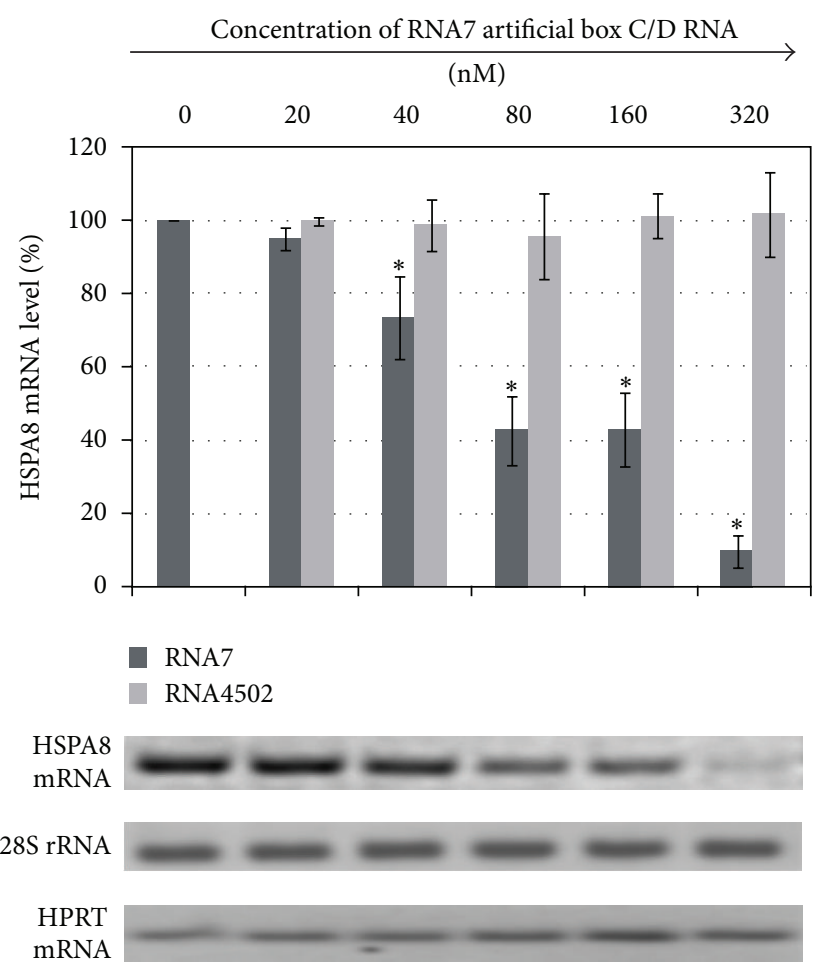

FIgURE 4: Level of HSPA8 mRNA after RNA7 transfection into MCF-7 cells. The indicated concentrations of RNA7 were transfected into cells using Lipofectamine, and the cells were cultured for $21 \mathrm{~h}$. Control cells ( $0 \mathrm{nM}$ RNA7) were incubated with Lipofectamine only. The error bars represent standard deviations. The asterisks $\left({ }^{*}\right)$ indicate significant difference from control $(P<0.05)$.

above the same set of artificial box C/D RNA induced the most conspicuous influence on the level of HSPA8 mRNAs (Figure 3).

When RNA concentration was increased up to $160 \mathrm{nM}$ correlation between HSPA8 mRNA suppression effect and MCF-7 viability reduction was not observed (Figure 6). Moreover, as it is seen from Figures 2 and 6, RNA4502 did not induce appreciable suppression of the HSPA8 mRNA expression but nevertheless reduced MCF-7 viability (by 22\%, $35 \%$, and $37 \%$ at $40 \mathrm{nM}, 80 \mathrm{nM}$, and $160 \mathrm{nM}$, resp., Figure 6). These facts suggested that in addition to the decrease in the HSPA 8 mRNA level there was some accessory process affecting the viability of transfected human cells. It is most likely that accompanying process induced in response to artificial box C/D RNA was activation of the innate immune system in transfected cells. The combination of the genespecific knockdown and the activation of the interferon response system were reported earlier for a small interfering RNA-dependent regulation of gene expression [31-33].

3.5. Complementary Interaction with the Target Pre-mRNA is the Key Factor for the Knockdown Ability of Box C/D RNA Analogues. The questions of whether box C/D snoRNAs direct $2^{\prime}$-O-methylation during their interaction with premRNAs and whether modification of the target nucleotide is essential for the ability of the snoRNA to regulate gene

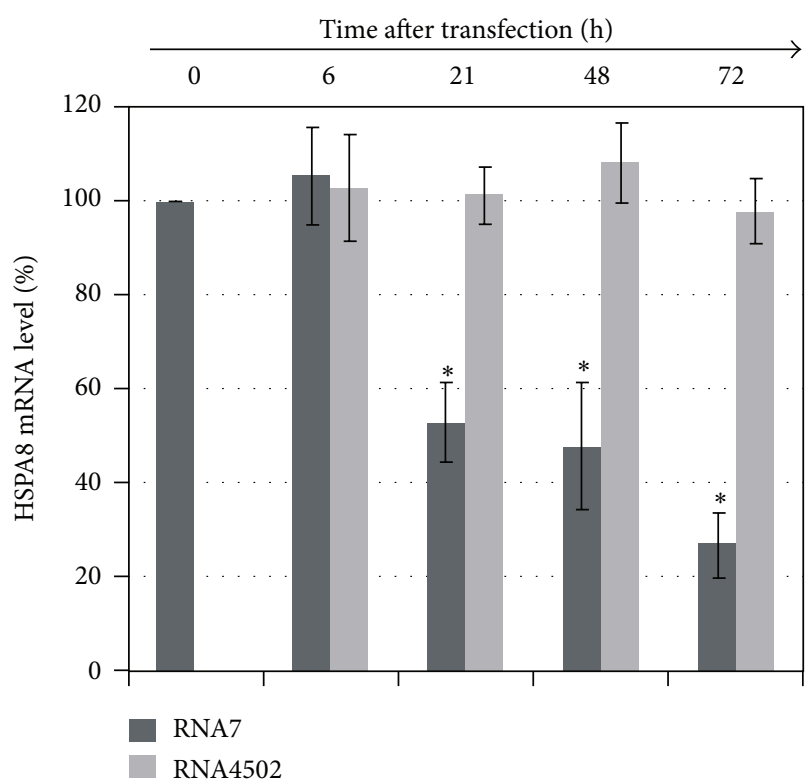

(a)

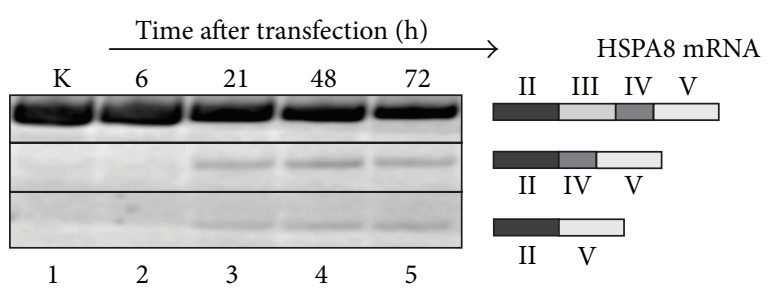

(b)

Figure 5: (a) Time course of the HSPA8 mRNA RNA level in MCF7 cells treated with artificial box C/D RNAs. RNA7 (160 nM) was transfected into cells using Lipofectamine, and then the cells were cultured for time intervals from $6 \mathrm{~h}$ to $72 \mathrm{~h}$. Control cells (indicated as " $0 \mathrm{~h}$ ") were incubated with Lipofectamine only. The error bars represent standard deviations. The asterisks $\left({ }^{*}\right)$ indicate significant difference from control $(P<0.05)$. (b) Splicing variants of HSPA8 pre-mRNA after transfection of artificial box C/D RNA targeted to the branch point adenosine into MCF-7 cells. RNA7 (160 nM) was transfected into cells using Lipofectamine. Cells were cultured for the time indicated. Control cells were incubated with Lipofectamine only. The schematic structures of splicing forms are represented on the left, with II, III, IV, and V corresponding to the numbers of HSPA 8 mRNA exons.

expression remain open. Classical methods to detect $2^{\prime}$ O-methylated RNA nucleotides do not reveal modified nucleotides in minor RNA forms such as pre-mRNA. At the same time, box D is known to be an essential element for snoRNA-guided 2'-O-methylation.

To study the participation of box D of artificial RNAs in pre-mRNA knockdown activity, we constructed U24 RNA analogues RNA7D* and RNA8D*, where both the box D and $\mathrm{D}^{\prime}$ CUGA sequences were replaced with AAAA (Figure 1(b)). RNA7D $^{*}$ and RNA8D* were targeted to the branch point adenosine in the second intron and the last nucleotide of the second exon of HSPA8 pre-mRNA, respectively. 


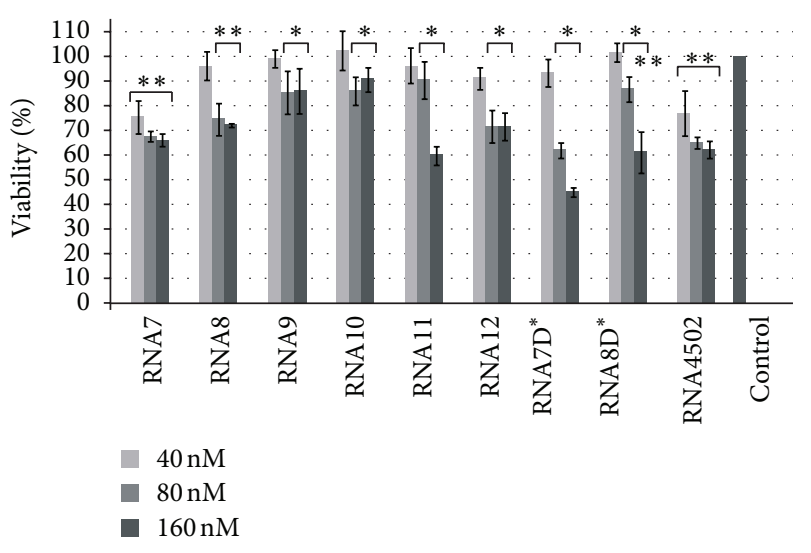

FIgURE 6: The effect of box C/D RNA analogues on the viability of human adenocarcinoma MCF-7 cells. Cells were transfected with $40 \mathrm{nM}, 80 \mathrm{nM}$, and $160 \mathrm{nM}$ artificial RNA (in complex with Lipofectamine) for $72 \mathrm{~h}$ and viability was analyzed by MTT assay. Control cells were incubated with Lipofectamine only. Data are presented as the mean of at least three separate experiments. The error bars represent standard deviations. The asterisks $\left({ }^{*}\right.$ or $\left.{ }^{* *}\right)$ indicate significant difference from control $\left({ }^{*} P<0.05\right.$ and ${ }^{* *} P<$ $0.01)$.

We found that transfection of RNA7D* or RNA8D* into human cells decreased the HSPA 8 mRNA level to $53 \pm 9.3 \%$ and $68 \pm 5.6 \%$, respectively (Figure 3 ). The decrease in targeted mRNA expression induced by RNAs with a mutated Dbox RNAs was practically the same as the decrease in expression caused by the nonmutated counterparts. This result indicated that the mutation of box D in artificial RNA did not abolish the HSPA8 mRNA suppression effect. Our results showed that neither the box D structural element (CUGA) nor the ability to direct $2^{\prime}$-O-methylation of nucleotides in target RNA were essential for the knockdown activity of artificial snoRNAs. Thus, complementary interaction with the target pre-mRNA was supposed to be the main pathway of artificial RNA influence on the target pre-mRNA maturation.

There are numerous RNA transcripts that are complementary to different pre-mRNAs in eukaryotic cells, but their functions are not well understood. Antisense transcripts could take part in a variety of vital processes including chromatin remodeling, regulation of transcription, posttranscriptional modifications, and intracellular transport [3437].

Our results suggest that posttranscriptional pre-mRNA maturation could be affected by artificial analogues of snoRNAs with antisense sequences that are complementary to premRNAs. Furthermore, our results suggest that the observed reduction of mRNA level occurs independently of the $\mathrm{D} / \mathrm{D}^{\prime}$ boxes of the analogues and thus independently of their potential ability to guide $2^{\prime}$-O-methylation of the target premRNA.

\section{Conclusion}

The study provides a novel tool to offer insights into the question of whether snoRNAs that are complementary to pre-mRNAs could affect the processes of target pre-mRNA maturation. Our results showed that transfection of human cells with artificial snoRNAs containing guide sequences directed to pre-mRNA could impair the splicing and affect overall mRNA isoform levels. Transfection with artificial snoRNAs decreased human cell viability, suggesting that snoRNA analogues are able to activate regulatory processes affecting vital cellular functions.

\section{Conflict of Interests}

The authors declare that they have no conflict of interests.

\section{Acknowledgments}

The work was supported by the Russian Ministry of Education and Science (14.132.21.1335), Russian Foundation for Basic Research Grants 10-04-01386-a and 10-04-01442-a, OPTEC Grant for support of young scientists. The authors thank Dr. Venyaminova A. G. (Institute of Chemical Biology and Fundamental Medicine SB RAS) for generously providing them with oligoribonucleotides. Thanks to Dr. Fomin A. S. (Institute of Chemical Biology and Fundamental Medicine SB RAS) for the synthesis $\left[\gamma^{32} \mathrm{P}\right]$ ATP used in this work.

\section{References}

[1] J. Cavaille, M. Nicoloso, and J. P. Bachellerie, "Targeted ribose methylation of RNA in vivo directed by tailored antisense RNA guides," Nature, vol. 383, no. 6602, pp. 732-735, 1996.

[2] K. T. Tycowski, C. M. Smith, M. D. Shu, and J. A. Steitz, "A small nucleolar RNA requirement for site-specific ribose methylation of rRNA in Xenopus," Proceedings of the National Academy of Sciences of the United States of America, vol. 93, no. 25, pp. 14480-14485, 1996.

[3] P. Ganot, M. L. Bortolin, and T. Kiss, "Site-specific pseudouridine formation in preribosomal RNA is guided by small nucleolar RNAs," Cell, vol. 89, no. 5, pp. 799-809, 1997.

[4] Z. Kiss-László, Y. Henry, and T. Kiss, "Sequence and structural elements of methylation guide snoRNAs essential for sitespecific ribose methylation of pre-rRNA," The EMBO Journal, vol. 17, no. 3, pp. 797-807, 1998.

[5] N. J. Watkins, V. Ségault, B. Charpentier et al., "A common core RNP structure shared between the small nucleoar box C/D RNPs and the spliceosomal U4 snRNP," Cell, vol. 103, no. 3, pp. 457-466, 2000.

[6] D. R. Newman, J. F. Kuhn, G. M. Shanab, and E. S. Maxwell, "Box C/D snoRNA-associated proteins: two pairs of evolutionarily ancient proteins and possible links to replication and transcription," RNA, vol. 6, no. 6, pp. 861-879, 2000.

[7] N. M. Cahill, K. Friend, W. Speckmann et al., "Site-specific cross-linking analyses reveal an asymmetric protein distribution for a box C/D snoRNP," The EMBO Journal, vol. 21, no. 14, pp. 3816-3828, 2002.

[8] A. Hüttenhofer, M. Kiefmann, S. Meier-Ewert et al., "RNomics: an experimental approach that identifies 201 candidates for novel, small, non-messenger RNAs in mouse," The EMBO Journal, vol. 20, no. 11, pp. 2943-2953, 2001.

[9] J. P. Bachellerie, J. Cavaille, and A. Huttenhofer, “The expanding snoRNA world," Biochimie, vol. 84, no. 8, pp. 775-790, 2002. 
[10] P. S. Bazeley, V. Shepelev, Z. Talebizadeh et al., "snoTARGET shows that human orphan snoRNA targets locate close to alternative splice junctions," Gene, vol. 408, no. 1-2, pp. 172-179, 2008.

[11] P. Vitali, E. Basyuk, E. Le Meur et al., "ADAR2-mediated editing of RNA substrates in the nucleolus is inhibited by C/D small nucleolar RNAs," The Journal of Cell Biology, vol. 169, no. 5, pp. 745-753, 2005.

[12] S. Kishore and S. Stamm, "The snoRNA HBII-52 regulates alternative splicing of the serotonin receptor 2C," Science, vol. 311, no. 5758, pp. 230-232, 2006.

[13] M. Ono, K. Yamada, F. Avolio et al., "Analysis of human small nucleolar RNAs (snoRNA) and the development of snoRNA modulator of gene expression vectors," Molecular Biology of the Cell, vol. 21, no. 9, pp. 1569-1584, 2010.

[14] S. Lindquist and E. A. Craig, "The heat shock proteins," Annual Review of Genetics, vol. 22, pp. 631-677, 1988.

[15] J. Nylandsted, M. Gyrd-Hansen, A. Danielewich et al., "Heat shock protein 70 promotes cell survival by inhibiting lysosomal membrane permeabilization," Journal of Experimental Medicine, vol. 200, no. 4, pp. 425-435, 2004.

[16] M. Rohde, M. Daugaard, M. H. Jensen, K. Helin, J. Nylandsted, and M. Jäättelä, "Members of the heat-shock protein 70 family promote cancer cell growth by distinct mechanisms," Genes and Development, vol. 19, no. 5, pp. 570-582, 2005.

[17] N. F. Krynetskaia, M. S. Phadke, S. H. Jadhav, and E. Y. Krynetskiy, "Chromatin-associated proteins HMGB1/2 and PDIA3 trigger cellular response to chemotherapy-induced DNA damage," Molecular Cancer Therapeutics, vol. 8, no. 4, pp. 864-872, 2009.

[18] Y. Q. Wei, X. Zhao, Y. Kariya, K. Teshigawara, and A. Uchida, "Inhibition of proliferation and induction of apoptosis by abrogation of heat-shock protein (HSP) 70 expression in tumor cells," Cancer Immunology, Immunotherapy, vol. 40, no. 2, pp. 73-78, 1995.

[19] J. Nylandsted, W. Wick, U. A. Hirt et al., "Eradication of glioblastoma, and breast and colon carcinoma xenografts by Hsp70 depletion," Cancer Research, vol. 62, no. 24, pp. 71397142, 2002.

[20] Z. G. Zhao and W. L. Shen, "Heat shock protein 70 antisense oligonucleotide inhibits cell growth and induces apoptosis in human gastric cancer cell line SGC-7901," World Journal of Gastroenterology, vol. 11, no. 1, pp. 73-78, 2005.

[21] G. A. Stepanov, D. V. Semenov, E. V. Kuligina et al., "Analogues of artificial human box C/D small nucleolar RNA as regulators of alternative splicing of a pre-mRNA target," Acta Naturae, vol. 4, no. 1, pp. 32-41, 2012.

[22] G. Kol, G. Lev-Maor, and G. Ast, "Human-mouse comparative analysis reveals that branch-site plasticity contributes to splicing regulation," Human Molecular Genetics, vol. 14, no. 11, pp. 15591568, 2005.

[23] Z. Kiss-László, Y. Henry, J. P. Bachellerie, M. Caizergues-Ferrer, and T. Kiss, "Site-specific ribose methylation of preribosomal RNA: a novel function for small nucleolar RNAs," Cell, vol. 85, no. 7, pp. 1077-1088, 1996.

[24] L. H. Qu, Y. Henry, M. Nicoloso et al., "U24, a novel intronencoded small nucleolar RNA with two $12 \mathrm{nt}$ long, phylogenetically conserved complementarities to $28 \mathrm{~S}$ rRNA," Nucleic Acids Research, vol. 23, no. 14, pp. 2669-2676, 1995.

[25] D. M. Graifer, G. G. Karpova, and D. G. Knorre, "Location of template on the human ribosome as revealed from data on cross-linking with reactive mRNA analogs," Biochemistry, vol. 66, no. 6, pp. 585-602, 2001.

[26] E. Nagy and L. E. Maquat, "A rule for termination-codon position within intron-containing genes: when nonsense affects RNA abundance," Trends in Biochemical Sciences, vol. 23, no. 6, pp. 198-199, 1998.

[27] J. Weischenfeldt, J. Waage, G. Tian et al., "Mammalian tissues defective in nonsense-mediated mRNA decay display highly aberrant splicing patterns," Genome Biology, vol. 13, no. 5, p. R35, 2012.

[28] S. Vasudevan and S. W. Peltz, "Nuclear mRNA surveillance," Current Opinion in Cell Biology, vol. 15, no. 3, pp. 332-337, 2003.

[29] P. Sazani and R. Kole, "Therapeutic potential of antisense oligonucleotides as modulators of alternative splicing," The Journal of Clinical Investigation, vol. 112, no. 4, pp. 481-486, 2003.

[30] J. A. Bauman and R. Kole, "Modulation of RNA splicing as a potential treatment for cancer," Bioengineered Bugs, vol. 2, no. 3, pp. 125-128, 2011.

[31] M. Schlee, V. Hornung, and G. Hartmann, "siRNA and isRNA: two edges of one sword," Molecular Therapy, vol. 14, no. 4, pp. 463-470, 2006.

[32] H. Poeck, R. Besch, C. Maihoefer et al., " 5 ' -triphosphate-siRNA: turning gene silencing and Rig-I activation against melanoma," Nature Medicine, vol. 14, no. 11, pp. 1256-1263, 2008.

[33] R. Kenworthy, D. Lambert, F. Yang et al., "Short-hairpin RNAs delivered by lentiviral vector transduction trigger RIGI-mediated IFN activation," Nucleic Acids Research, vol. 37, no. 19, pp. 6587-6599, 2009.

[34] M. U. Kaikkonen, M. T. Y. Lam, and C. K. Glass, "Noncoding RNAs as regulators of gene expression and epigenetics," Cardiovascular Research, vol. 90, no. 3, pp. 430-440, 2011.

[35] R. Knee and P. R. Murphy, "Regulation of gene expression by natural antisense RNA transcripts," Neurochemistry International, vol. 31, no. 3, pp. 379-392, 1997.

[36] J. Han, D. Kim, and K. V. Morris, "Promoter-associated RNA is required for RNA-directed transcriptional gene silencing in human cells," Proceedings of the National Academy of Sciences of the United States of America, vol. 104, no. 30, pp. 12422-12427, 2007.

[37] M. Nishizawa, T. Okumura, Y. Ikeya, and T. Kimura, "Regulation of inducible gene expression by natural antisense transcripts," Frontiers in Bioscience, vol. 1, no. 17, pp. 938-958, 2012. 

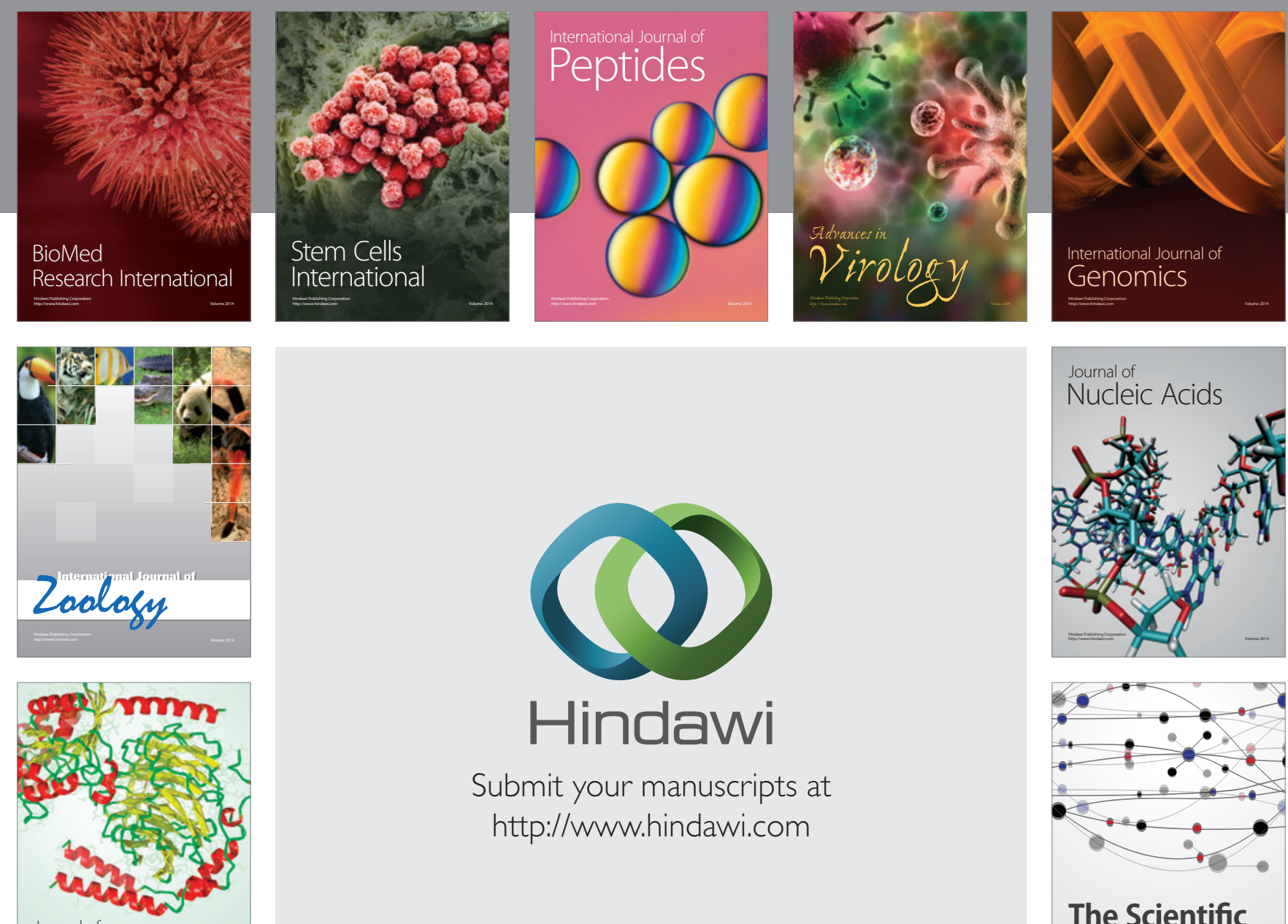

Submit your manuscripts at

http://www.hindawi.com

Journal of
Signal Transduction
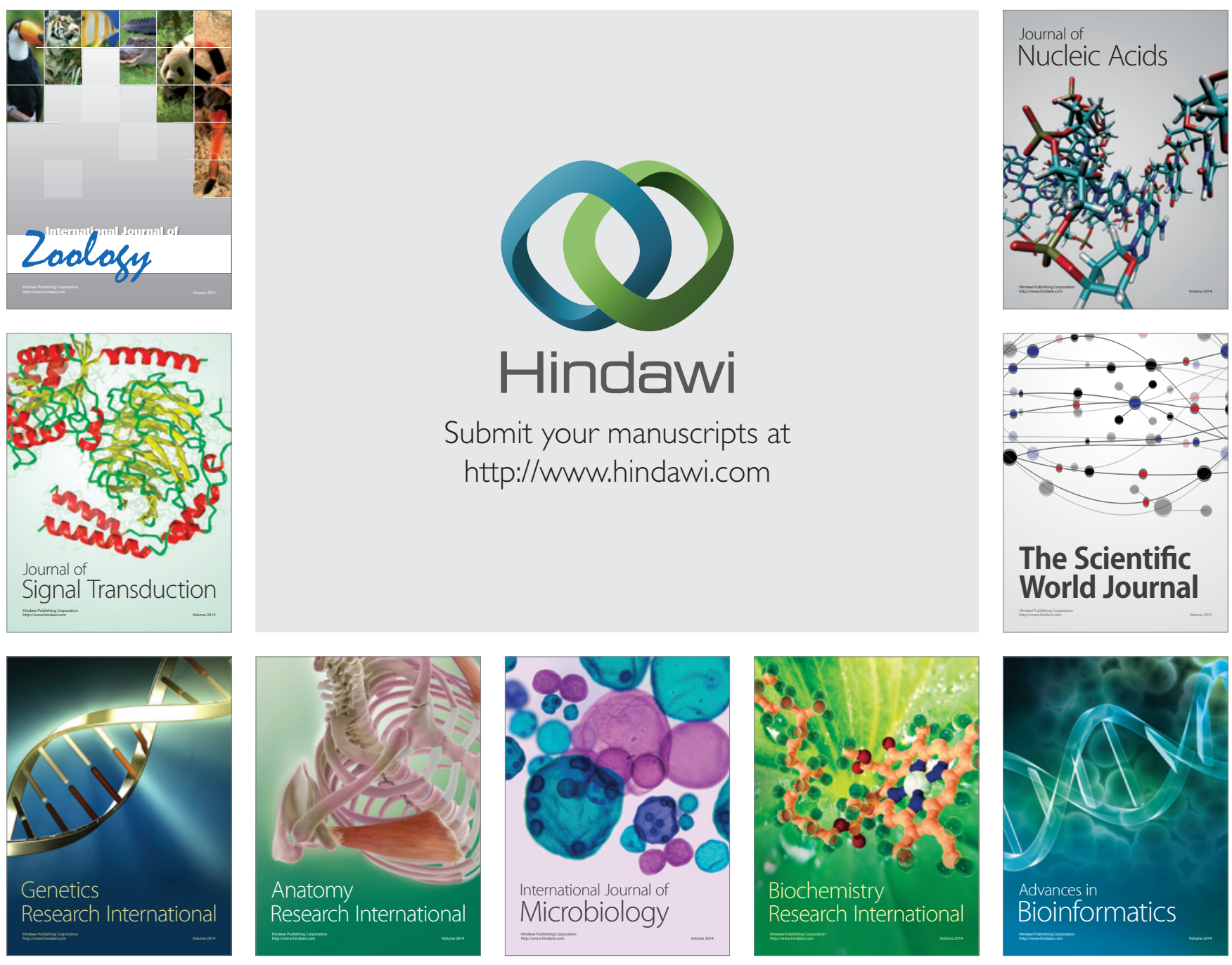

The Scientific World Journal
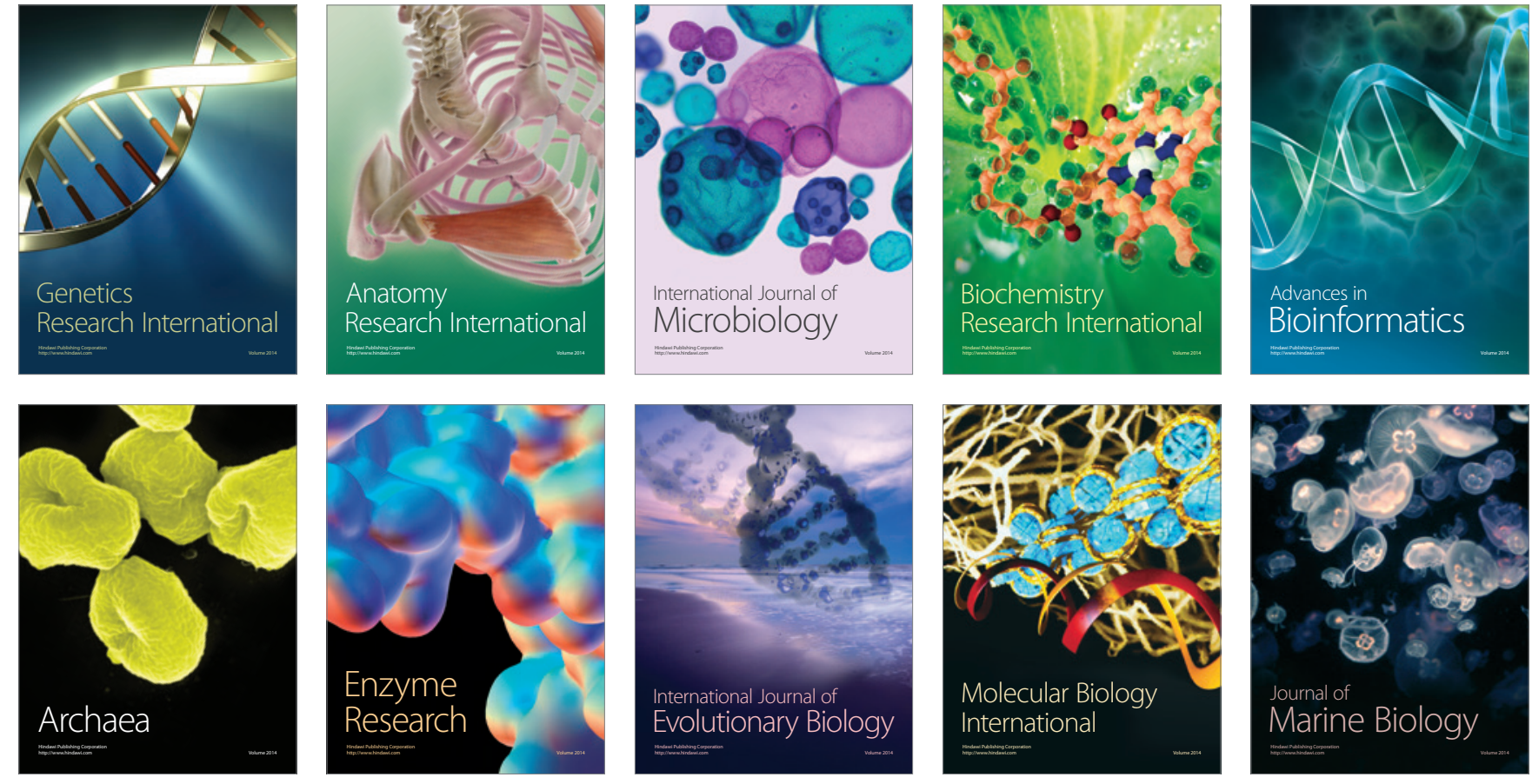